\title{
Desafios para a Formação do Neuropsicólogo Clínico no Brasil
}

\author{
Ana Paula Almeida de Pereira* \\ Universidade Federal do Paraná, Curitiba, PR, Brasil
}

\begin{abstract}
RESUMO
A Neuropsicologia Clínica é um campo de trabalho recente no Brasil, no entanto, existe uma forte demanda no mercado por profissionais qualificados. Embora o Conselho Federal de Psicologia já tenha esboçado este campo como uma especialidade da formação em Psicologia, ainda não existe um consenso sobre como deve ser delineada a formação desse profissional. O presente artigo busca, através de uma análise histórica da área, delimitar esse campo de conhecimento para, então, observando os processos de formação do neuropsicólogo clínico em outros países mais avançados nesta discussão, traçar um breve perfil das competências, atitudes e comportamentos desse profissional. Finalmente, apontar os desafios que a formação do neuropsicólogo clinico representa no contexto brasileiro.
\end{abstract}

Palavras-chave: neuropsicologia clínica; formação profissional; Brasil.

\begin{abstract}
Challenges for the Professional Education of the Clinical Neuropsychologist in Brazil

Clinical Neuropsychology is a fairly recent field of work in Brazil, however, there is a growing demand for professionals within this specialty. Though the Federal Council of Psychology has already delineated this field as a possible specialty after professional graduate training, the requirements for such training are still unclear. The present article seeks to build this field's framework by pointing out the competencies attitudes and behaviors of the Clinical Neurospychologists and by investigating historical aspects and the training requirements established by other countries which are more advanced in the debate. Finally, the major challenges for providing training in clinical neuropsychology are pointed out.
\end{abstract}

Keywords: clinical neuropsychology, professional training, Brasil.

A Neuropsicologia definiu-se como um campo de estudo interdisciplinar que busca compreender a relação entre o comportamento e o cérebro, no qual conhecimentos de diversas áreas da ciência contribuem para sua construção. Esta peculiaridade representa um desafio para a formação em Neuropsicologia, especialmente, para o profissional que irá se dedicar à Neuropsicologia Clínica. O presente artigo propõe contribuir para a reflexão sobre a formação em Neuropsicologia Clínica no Brasil como um campo de atuação do psicólogo.

Um importante debate tem sido travado com o intuito de delimitar as especificidades do trabalho do psicólogo especialista em Neuropsicologia sem, po- rém, ignorar que os conhecimentos provenientes de pesquisas em Neuropsicologia são construídos através do estudo em diversas áreas da ciência (por exemplo, neurologia, psicofarmacologia, psicobiologia, linguística e psicologia cognitiva). O trabalho do neuropsicólogo clinico caracteriza-se tanto por uma formação interdisciplinar em neurociências e psicologia quanto por buscar uma pratica clinica voltada para os objetos de intervenção e estudo da Psicologia Clinica. Assim, por exemplo, o campo de estudo da Neuropsicologia da memória integra informações provenientes de disciplinas diversas como biologia celular, neurofisiologia e neurologia, ou seja, a Neuropsicologia faz parte da grande área denominada Neurociências, porém, apresenta especificidades importantes que a discrimi- 
nam em sua atuação clínica. Outro exemplo, o psicólogo, especialista em Neuropsicologia Clínica, ao intervir junto a um cliente com problemas de memória deve se utilizar deste conhecimento interdisciplinar mantendo o foco em objetivos característicos de sua especialidade como a facilitação das relações sociais e familiares através de estratégias compensatórias para reabilitação da memória.

O debate sobre a formação em Neuropsicologia, frequentemente, negligencia a diferença entre a necessidade de se divulgar os conhecimentos em Neuropsicologia para diversos profissionais e a formação específica do profissional psicólogo que se propõe a prática da Neuropsicologia Clínica. Talvez, isto ocorra devido ao fato que, no país, ainda existam poucas equipes multiprofissionais que efetivamente trabalham de modo interdisciplinar nos diversos contextos, ou ainda, devido ao conhecimento incipiente sobre as características específicas das funções do Neuropsicólogo Clínico.

É consenso que se deve fomentar a divulgação do conhecimento em Neuropsicologia junto a profissionais como o fonoaudiólogo, o fisioterapeuta, o terapeuta ocupacional, o educador, ou o psiquiatra, para citar alguns dos profissionais que trabalham em diferentes contextos de reabilitação e inclusão de pessoas com distúrbios neuropsicológicos. Esta necessidade decorre pelo fato que tal conhecimento pode tanto tornar o trabalho individual de cada um dos profissionais da equipe mais eficaz, quanto facilitar a comunicação entre os profissionais envolvidos e a elaboração de um plano interdisciplinar de trabalho. Neste plano interdisciplinar existiriam intervenções específicas determinadas pela formação profissional e os objetivos característicos de cada uma destas áreas profissionais. Deste modo, o terapeuta ocupacional, por exemplo, ao fazer uma avaliação dentro do contexto da equipe, se utilizaria de instrumentos avaliativos com enfoque na compreensão das diferentes atividades humanas desempenhadas pelo cliente e proporia um plano de intervenção para organizar seu cotidiano (dia a dia), possibilitando-lhe melhor qualidade de vida. A prática profissional é pautada por um procedimento específico que leva a um objetivo em comum entre os profissionais da equipe: melhoria da qualidade de vida. Assim, cada um destes profissionais da equipe interdisciplinar apresentaria procedimentos próprios derivados de seus objetivos específicos, mas interconectados através de metas interdisciplinares.

A Neuropsicologia, como campo de conhecimento deve estar ao alcance de qualquer profissional que se interesse pelo tema e, tal tarefa, pode ser realizada através de diferentes recursos da comunidade como universidades, sociedades profissionais, ou ainda, associações acadêmicas. O profissional busca continuidade em sua formação através de livros, eventos científicos e cursos de capacitação continuada. No entanto, os requisitos necessários para a formação do neuropsicólogo clínico requerem uma reflexão maior. Primeiramente, para proteger o cliente que busca serviços profissionais de neuropsicologia de qualidade e, em segundo lugar, para que os próprios profissionais em formação possam fazer suas escolhas de capacitação continuada é preciso que a formação necessária para atuar como neuropsicólogo clínico seja claramente divulgada.

O presente artigo propõe uma delimitação das competências e habilidades do psicólogo especialista em Neuropsicologia Clínica em relação a outros profissionais da área de Neurociências. Esta delimitação será fundamentada através de uma breve apresentação histórica sobre a formação profissional do neuropsicólogo clínico e da compreensão de alguns dos requisitos teóricos e metodológicas necessários para prática da Neuropsicologia Clínica.

\section{A história da formação em Neuropsicologia Clínica}

O campo da Neuropsicologia caracteriza-se, desde sua origem, por apresentar elementos provenientes de estudos multidisciplinares experimentais e clínicos. Sendo assim, a Neuropsicologia engloba uma diversidade epistemológica e metodológica que pode ter fomentado controvérsias sobre a formação e a prática profissional nesta área recentemente criada no Brasil. Kristensen, Almeida e Gomes (2001) buscaram descrever o surgimento da Neurociência Cognitiva a partir do estudo das lesões cerebrais e seus efeitos na cognição e no comportamento humano. Estes autores discutiram ainda a importância de diferentes metodologias na construção do conhecimento na área: estudo de caso e estudo de grupos, tarefas cognitivas e mais recentemente, as técnicas de neuroimagem. A Neuropsicologia Clínica seguiu um caminho semelhante. 
Frequentemente, os primórdios da Neuropsicologia são relacionados com os estudos da afasia. Segundo Manning (2005), a Neuropsicologia Clínica nasceu na metade do século XIX com o estudo de caso de afasia registrado por Paul Broca um médico e pesquisador interessado em neuroanatomia (1861). No século XX, Brenda Milner, psicóloga e pesquisadora, acompanhou durante anos o paciente H.M. após uma neurocirurgia que provocou um déficit profundo nas suas funções de aprendizagem e memória. Desde então, diversos casos de pacientes com lesão foram relatados e os distúrbios psicológicos derivados do insulto ao sistema nervoso central, cuidadosamente, descritos. Pode-se dizer que parte importante do conhecimento atual em Neuropsicologia foi construído através dos estudos de casos, os quais, muitas vezes, foram acompanhados longitudinalmente pelo pesquisador/profissional (Wilson, 1999). Na área de reabilitação neuropsicológica, estudos de caso único com desenho experimental têm sido apontada como um método importante para compreender a relação entre as intervenções clínicas e suas consequências (Tate, Perdices, Rosenkoetter, Wakim, Godbee, Togher, \& McDonald, 2013).

Simultaneamente, a influência dos estudos com delineamentos experimentais também pode ser observada. Desde W. Wundt e J. Watson, a tradição experimental influencia as pesquisas realizadas em diferentes campos da Psicologia, inclusive a Neuropsicologia. O importante papel da Psicometria como área fundamental para a construção da prática da Avaliação Neuropsicológica também deve ser destacado pois reforçou a tradição que busca medir e predizer comportamentos e características psicológicas.

Um dos periódicos mais importantes e antigos da área de Neuropsicologia, em seu título já claramente demonstra a importância dos métodos de delineamento clínico e experimental para a área: o Journal of Clinical and Experimental Neuropsychology encontra-se em seu $36^{\circ}$ ano de existência e revela a busca de diálogo interdisciplinar na área. Pode-se dizer que estudos clínicos fomentaram a organização de pesquisas experimentais e estes, por sua vez, influenciaram a pesquisa e a prática clínica. A pesquisa em Neuropsicologia caracteriza-se assim, não apenas pela busca do conhecimento e a criação de modelos teóricos sobre a relação cérebro e cognição/comportamento mas, igualmente, por fundamentos que possibilitem uma prática clínica.

Observam-se pelo menos dois sinais do crescimento da área de Neuropsicologia Clínica: o aumento de publicações científicas específicas para o tópico e ampliação do número de organizações profissionais fundadas em diferentes países que buscam fomentar a pesquisa aplicada e a formação profissional. Nos Estados Unidos da América, as primeiras organizações profissionais congregando profissionais de áreas variadas surgiram nos últimos 60 anos. No Brasil, diferentes organizações profissionais começaram suas atividades a partir do final do século passado.

A fundação da Neuropsicologia como um campo de pesquisa e de prática profissional, formalmente, ocorreu primeiro nos Estados Unidos. Embora pesquisadores e profissionais de vários países já trabalhassem em Neuropsicologia. Diversos marcos podem ser ressaltados que referendam esta afirmativa, mas o principal evento parece ter sido a criação da International Neuropsychological Society (INS), em 1967. Esta associação constituiu-se, inicialmente, de pesquisadores em diferentes áreas das neurociências, ligados principalmente à fisiologia e à psicobiologia, que buscavam um espaço formal de discussão e integração das pesquisas sobre a relação cérebro e comportamento. Atualmente, a INS conta com mais de 4500 membros, organiza duas reuniões anuais em diferentes países do mundo e oferece sistematicamente cursos de formação continuada. Nestas reuniões, o equilíbrio entre as apresentações da pesquisa experimental e da pesquisa clínica permanece até hoje (http://www. the-ins.org/society-info).

Apenas em 1980, a Associação de Psicologia Americana (American Psychological Association) criou uma divisão de Neuropsicologia Clínica (APA Division 40). Esta diferença temporal pode indicar que o trabalho inicial na Neuropsicologia, enquanto campo de atuação formal, ocorreu dentro do contexto da academia, dos hospitais universitários e, principalmente, laboratórios. A INS teve um papel importante para liderar um grupo de trabalho junto a APA, que em 1987, definiu pela primeira vez consensualmente as atribuições do neuropsicólogo clínico. Dez anos depois, em 1997, na Conferência de Houston, publicou as diretrizes americanas que fundamentam a formação de neuropsicólogos clínicos neste país (http://www. 
apa.org/ed/graduate/specialize/neuro.aspx). Deste modo, no contexto norte-americano, a formação profissional em Neuropsicologia Clínica passou a ser claramente distinta da formação em Neuropsicologia Experimental, em Neurociência Cognitiva, ou em Psicobiologia, uma vez que, pressupõe habilidades clínicas de intervenção, caracterizando-se como uma especialidade da atuação profissional do psicólogo. Deste modo, diversos programas de Neuropsicologia Clínica encontram-se ligados a departamentos e programas de pós-graduação de Psicologia Clínica nos Estados Unidos e na Europa. Esta especificidade deve ser considerada nas discussões brasileiras sobre a especialidade em Neuropsicologia Clínica como área de atuação profissional fundamentada na relação entre a psicologia e as neurociências.

Em 2013, o site da APA listava um total de 38 programas credenciados para formação de Neuropsicólogos Clínicos. Nos Estados Unidos, para se tornar Neuropsicólogo Clínico, deve-se fazer quatro anos do curso de graduação em Psicologia e, posteriormente, um Programa de quatro a cinco anos de Doutorado e mais um ano de estágio pós-doutoral e dois anos de residência supervisionada na área, ou seja, requer onze anos de formação.

Os requisitos para a formação em Neuropsicologia Clínica diferem entre países. Na Austrália, a formação ocorre após três anos de curso de graduação em Psicologia, e um ano de estágio, e em mais dois anos de Mestrado ou três anos de Doutorado em Neuropsicologia Clinica e apenas cerca de cinco universidades oferecem Programas na área. No Reino Unido, é necessária formação de três anos de curso de graduação em Psicologia, três anos de Doutorado em Psicologia Clínica ou Educacional e, posteriormente, mais um ano de Mestrado ou pós-graduação específica em Neuropsicologia Clínica em uma das poucas universidades que oferecem esta formação (quatro no momento da redação deste artigo) (British Psychological Society, 2013). Enfim, nesses países, a formação em Neuropsicologia Clínica foi instituída recentemente de modo formal e já apresenta algumas coincidências importantes:

1. A formação se difere da formação em Neuropsicologia Experimental ou outras áreas das Neurociências, pois objetiva primordialmente a capacitação do psicólogo para o atendimento clínico.

2. Além da formação em pesquisa científica, os profissionais em treinamento passam por experiências clínicas supervisionadas extensas e específicas em contextos de Neuropsicologia Clínica.

3. Todos os sistemas de formação reconhecem a importância da graduação em Psicologia como alicerce para a capacitação pós-graduada em Neuropsicologia Clínica.

4. As associações profissionais e a academia exercem um papel ativo na elaboração de diretrizes para a formação profissional do Neuropsicólogo assim como, no modo em que sua prática deve ser validada.

Cada uma dessas semelhanças entre a formação em diferentes países será analisada na próxima sessão considerando-se as competências que buscam desenvolver no neuropsicólogo com formação clínica.

No Brasil, a forte influência destas duas vertentes, experimental e clínica, também pode ser observada na história da Neuropsicologia brasileira. Em 1988, a Sociedade Brasileira de Neuropsicologia formou-se através da iniciativa de diferentes grupos ligados a instituições de pesquisa e preocupados com a relação cérebro, cognição e comportamento. Dois neurologistas ligados à academia tiveram papel essencial em congregar tais grupos: o Dr. Jayme Antunes Maciel Jr (UNICAMP) e o Dr. Norberto Rodrigues (PUC-SP) (http://sbnp.com.br/site/index.php/asbnp/historia/).

Esta organização, tal qual a INS, fomenta a interação entre os diversos profissionais das neurociências e de outras áreas e tem papel importante na divulgação do conhecimento científico da Neuropsicologia através de eventos científicos, cursos de formação continuada e publicações.

Em 2009, o Instituto Brasileiro de Neuropsicologia e Comportamento (IBNeC) foi criado para refletir sobre a interação da Psicologia com as Neurociências e fomentar a interação entre pesquisa básica e aplicada. Esta associação científica congrega profissionais de diferentes áreas e tem caráter multidisciplinar. Seguindo um dos principais propósitos de sua criação preocupa-se especialmente em participar do debate 
junto ao Conselho Regional de Psicologia eoutras organizações profissionais e científicas sobre a formação do Neuropsicólogo Clínico.

O Conselho Federal de Psicologia reconhece que o profissional especialista em Neuropsicologia utiliza-se de "conhecimentos teóricos angariados pelas neurociências e pela prática clínica, com metodologia estabelecida experimental ou clinicamente" (Conselho Federal de Psicologia, 2004). Porém, a resolução do CRP carece de uma delimitação clara e detalhada sobre a formação profissional do Neuropsicólogo que irá atuar no campo da clínica. Este debate ainda está em curso no Brasil. No presente artigo, buscou-se apresentar alguns dos principais marcos do conhecimento clínico da Neuropsicologia que, atualmente, constitui parte das competências e habilidades específicas do neuropsicólogo clínico.

\section{Conhecimentos e habilidades do neuropsicólogo clínico}

Os conhecimentos da Neuropsicologia, compartilhados entre diversos profissionais, fundamentam a prática do neuropsicólogo clínico, no entanto, tal prática apresenta características que requerem uma formação específica (Lamberty \& Nelson, 2012). A visão histórica de como o campo da Neuropsicologia Clínica se constituiu auxilia na compreensão destas características específicas.

Desde 2010, a Organização Mundial da Saúde (OMS) incentiva a educação interdisciplinar pois sabe--se que os serviços profissionais da área da saúde quando promovem a colaboração interdisciplinar tornam-se mais eficazes. No entanto, poucos cursos de graduação ou pós-graduação no Brasil propiciam em seus currículos as habilidades para este trabalho colaborativo, o que dificulta sobremaneira sua implantação na prática profissional. Este documento recomenda uma série de ações em busca da educação interdisciplinar e afirma que esta se fundamenta em um currículo que une as atividades dos estudantes aos resultados esperados. Para tanto, o aprendiz deve saber "o que fazer (conhecimento), como aplicar seu conhecimento (habilidade) e quando aplicá-lo seguindo as diretrizes éticas (atitudes e comportamento)" (tradução livre da autora, OMS, 2010). A formação em Neuropsicologia Clínica, sem dúvida, é um importante exemplo de capacitação em que as habilidades interdisciplinares devem ser cultivadas.

A formação em neuropsicologia é essencialmente interdisciplinar e a prática da Neuropsicologia Clínica torna-se enriquecida e mais eficaz no contexto da equipe multiprofissional. Consequentemente, tal formação deve tornar-se um exemplar destes parâmetros. Deste modo, faz-se necessário delimitar quais os conhecimentos e as habilidades necessários para formar o neuropsicólogo clínico sem perder de perspectiva sua interdisciplinaridade.

\section{Os conhecimentos}

Dentro do campo da Neuropsicologia, o conhecimento das bases neuroanatomo-fisiológicas do sistema nervoso central (SNC) é essencial, assim como para todos os profissionais que trabalham no campo das Neurociências Clínicas. Uma breve observação dos princípios epistemológicos que norteiam a prática do neuropsicólogo clinico indica que o estudo sobre a construção da pessoa como sujeito influenciado por fatores biopsicossociais que se relacionam com capacidades e limitações neuropsicológicas e estão envolvidos na expressão de sua identidade, ocupam papel privilegiado na perspectiva deste profissional. O conhecimento proveniente da Psicologia Clinica e da Psicometria fundamentam a utilização de estratégias variadas de avaliação e intervenção psicológica no contexto de pessoas com déficits neuropsicológicos o que constrói uma prática baseada em evidências e teoricamente consistente. Enfim, o corpo de conhecimento necessário para a atuação do Neuropsicólogo Clínico difere daquele da formação em Neuropsicologia Experimental ou outras áreas das Neurociências à medida que se trata de uma ciência aplicada que objetiva primordialmente a capacitação do profissional psicólogo para o atendimento de clientes com dificuldades neuropsicológicas. Deste modo, é coerente que a formação ao nível de graduação em Psicologia, especialmente no Brasil, onde o psicólogo já se encontra habilitado para o trabalho clínico, torne-se alicerce para a capacitação teórico-prática pós-graduada em Neuropsicologia Clínica.

\section{As habilidades}

O neuropsicólogo clínico deve ser capaz de transferir seus conhecimentos sobre o funcionamento do 
SNC para uma prática clínica em psicologia baseada em evidências. Por exemplo, quando déficits mnemônicos afetam a memória autobiográfica de uma pessoa, o foco da intervenção permanece na pessoa (seus sentimentos, comportamentos e pensamentos característicos) enquanto as diversas estratégias de intervenção são programadas para contornar ou minorar tais déficits no seu dia a dia e reconstruir sua identidade. Este enfoque funcional, compartilhado por vários profissionais da equipe de reabilitação/intervenção e fomentado pelos parâmetros da Classificação Internacional de Funcionalidade, Incapacidade e Saúde (Organização Mundial de Saúde, 2001) possibilita tanto o delineamento de intervenções com objetivos comuns entre os membros da equipe quanto à especificidade que a colaboração entre cada um deles pode proporcionar. Assim, outro exemplo pode ser apresentado: utilizar-se de um mesmo instrumento de trabalho ou atividade, como o brinquedo e o jogo, não deve ser restrito a um profissional da equipe, de fato, todos os profissionais que trabalham com crianças devem ter familiaridade em adaptar suas intervenções a utilização de estratégias motivadoras ao cliente. Deste modo, o que determina a especificidade da intervenção de um profissional não é o instrumento eleito como parte da estratégia de intervenção, no exemplo - o brinquedo/jogo - mas sim o objetivo específico e as estratégias utilizadas durante a intervenção.

Portanto, as habilidades para fomentar o trabalho e a comunicação interdisciplinar e para delimitar as responsabilidades e características profissionais devem ser adquiridas. A educação para a atuação interdisciplinar requer que os profissionais em treinamento passem por experiências clínicas supervisionadas extensas e específicas em contextos de Neuropsicologia Clínica. O modelo de Boulder (Peterson \& Park, 2005) pode contribuir para a construção dos programas educacionais brasileiros em Neuropsicologia Clínica pois apresenta vantagens importantes para guiar tais programas de formação uma vez que busca desenvolver uma prática profissional fundamentada em pesquisa científica e, simultaneamente, pode tornar o profissional um agente na construção do conhecimento. Esta relação entre teoria e prática pode fundamentar uma atuação ética e inovadora dos Neuropsicólogos.

\section{CONSIDERAÇÕES FINAIS}

Tanto a história da Neuropsicologia quanto as atuais diretrizes mundiais para a formação de profissionais da saúde indicam que a colaboração entre profissionais de diferentes especialidades é necessária e torna o serviço mais eficaz. Deste modo, a formação pósgraduada do profissional em Neuropsicologia Clínica deve incorporar os conhecimentos básicos obtidos durante o curso de graduação em psicologia, enfatizar as evidências da relação cérebro-compor-tamento, demonstrar a importância da intervenção psicológica para a plasticidade cerebral, facilitar a aquisição de conhecimentos sobre as estratégias compensatórias e o planejamento de intervenções neuropsicológicas, criar experiências supervisionadas onde os modos de comunicação compartilhada e as habilidades para a eleição de objetivos comuns e o trabalho interdisciplinar são efetivamente realizados entre os profissionais da equipe.

Em suma, sugere-se que tal como ocorreu em outros países, as associações profissionais e as instituições de ensino superior, principais responsáveis pela formação profissional no país, assumam não apenas o debate sobre a formação em Neuropsicologia mas também criem diretrizes claras que respondam aos desafios atuais para a formação profissional do Neuropsicólogo Clínico no Brasil apontados neste artigo: a) promover a formação voltada para a prática interdisciplinar; b) organizar as experiências de práticas supervisionadas em Neuropsicologia Clínica como parte integral da formação; c) incentivar que os cursos de graduação em Psicologia, especialmente aqueles com ênfase curricular nas áreas de intervenção clínica e de saúde, contemplem as disciplinas básicas para a formação em Neuropsicologia como Processos Psicológicos Básicos, Avaliação Psicológica ou Neuroanatomia; d) construir subsídios que viabilizem a avaliação dos programas de formação e seus currículos.

Finalmente, propõe-se a criação de grupos de trabalho regionais que apresentem suas propostas e reflexões em um debate nacional para que soluções para os desafios acima apontados sejam passíveis de serem acordadas e, posteriormente, adotadas. A busca da divulgação dos conhecimentos em Neuropsicologia deve ser combinada com o fomento de uma prática parcimoniosa e ética do Neuropsicólogo Clínico, e 
estão diretamente ligadas a excelência desta formação profissional.

\section{REFERÊNCIAS}

British Psychological Society (2013) Qualification in Clinical Neuropsychology (QiCN): Candidate Handbook, BPS, Leicester www.bps.org.uk/qicn.

Conselho Federal de Psicologia (2012). Resolução $\mathrm{n}^{\circ}$ 13/2007. Institui a Consolidação ao Titulo Profissional de Especialista. Obrtido de http://site.cfp.org.br/wpcontent/uploads/2006/01/resolucao2004_2.pdf.

INS-Division 40 Task Force on Education, Accreditation, and Credentialing. (1987). Report of the INS-Division 40 Task Force on Education, Accrditation, and Credentia-ling. Clinical Neuropsychologist, 1(1), 29-34.

Instituto Brasileiro de Neuropsicologia e Comportamento (2013). http://www.ibnec.org/sobre.html.

International Neuropsychological Society. (2013). Obtido de http://www.the-ins.org/society-info.

Kristensen, C. H., Almeida, R. M. M., Gomes, W. B. (2001). Desenvolvimento histórico e fundamentos metodológicos da neuropsicologia cognitiva. Psicologia: Reflexão e Crítica, 14(2), 259-274.

Lamberty, G. J., \& Nelson, N. W. (2012). Specialty competencies in clinical neuropsychology. Nova Iorque, EUA: Oxford.

Manning, L. (2005). A Neuropsicologia clínica: Uma abordagem cognitiva. Lisboa, Portugal: Instituto Piaget.
Organização Mundial da Saúde. (2010). Framework for Action on Interprofessional Education \& Collaborative Practice. Obtido de http://whqlibdoc.who.int/hq/2010/ WHO_HRH_HPN_10.3_eng.pdf.

Organização Mundial da Saúde. (2001). Classificação Internacional de Funcionalidade, Incapacidade e Saúde. Obtido de http://www.inr.pt/uploads/docs/cif/CIF_port_ \%202004.pdf.

Peterson, C., \& Park, N. (2005). The Enduring Value of the Boulder Model: "Upon This Rock We Will Build". Journal of Clinical Psychology, 61(9), 1147-1150.

Sociedade Brasileira de Neuropsicologia. (2013). Obtido de http://sbnp.com.br/site/index.php/asbnp/historia.

Tate, R., Perdices, M., Rosenkoetter, U., Wakim, D., Godbee, K., Togher, L., McDonald, S. (2013). Revision of a method quality rating scale for single-case experimental designs and n-of-1 trials: The 15-item Risk of Bias in N-of-1 Trials (RoBiNT) Scale. Neuropsychological Rehabilitation, 23(5), 619-638.

Wilson, B. A. (1999). Case studies in Neuropsychological Rehabilitation. Oxford: Oxford Press. 